\title{
Utilization of traditional Chinese medicine and the impact on medical burden of geriatric inpatients with chronic diseases
}

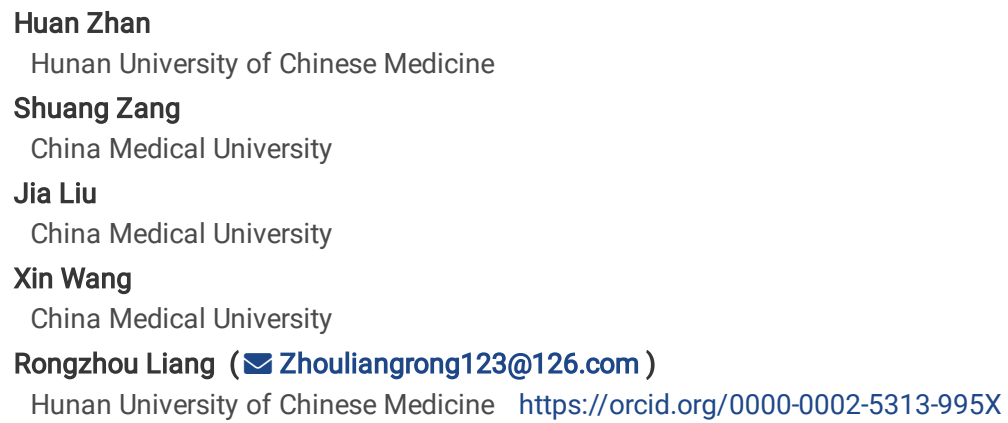

Research

Keywords: Geriatric, Chronic diseases, Traditional Chinese medicine, The proportion of expenses of traditional Chinese medicine to expenses of drugs, Total expenses of hospitalization, Out-of-pocket payments

Posted Date: October 20th, 2020

DOI: https://doi.org/10.21203/rs.3.rs-90764/v1

License: (c) (1) This work is licensed under a Creative Commons Attribution 4.0 International License. Read Full License 


\section{Abstract}

Background

The burden of chronic diseases seriously affects the health and quality of life of the geriatric. Many studies have pointed out that traditional Chinese medicine(TCM) can alleviate the problem of expensive medical treatment. It is unknown that how situation of the utilization of TCM in geriatric patients with chronic diseases and the impact of the use of TCM on their medical burden.

Methods

Through stratified multi-stage sampling method, 1336 institutions in Hunan Province of China were surveyed to obtain expenditure inpatient's of geriatric with chronic diseases in 2018. Possion regression model was used to analysis the utilization of TCM. Kolmogorov-Smirnov test was performed to observe the medical burden in TCM user' group and TCM nousers' group. The relationship between proportion of expenses of TCM to expenses of drugs(Ptcm) and medical burden were analyzed using Spearman's correlation analyses.

Results

In geriatric inpatients with chronic diseases, female ( $R R=1.009, R R 95 \% \mathrm{Cl}: 1.006-1.013)$, patients who have not undergone surgery $(R R=1.246, R R 95 \% C l: 1.237-$ 1.255), the elderly and the insured patients were more likely to use TCM for treatment. The distribution of Totalexp in the TCM users group and the TCM nousers group was different in various types of chronic diseases (all $p<0.001$ ). For some types of chronic diseases, the Ptcm has a negative correlation with

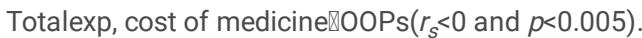

Conclusion

There were differences in the utilization of TCM treatment of geriatric patients with chronic diseases in terms of gender, age, institution and insurance status. Treatment mainly used TCM for geriatric chronic diseases of respiratory system, digestive system, circulatory system, ear and neurological has obvious costbenefit advantages, and conducive to reducing the burden of patients.

\section{Background}

As of the end of 2019, Chinese population aged 60 and over was 253.88 million, and population aged 65 and older was 176.03 million, accounting for $18.1 \%$ and $12.6 \%$ of the total population respectively[1]. According to the United Nations standard for aging society (the proportion of the total population $60 \mathrm{y}$ and over , $65 \mathrm{y}$ and over reached $10 \%$ and $7 \%$ respectively)[2], China has a deeper ageing. Due to the decline of body function and the long-term accumulation of disease factors[3], the elderly population has a higher prevalence and medical service needs than other age groups[4]. Zheng et al's research shown that the repeated hospitalization rate of geriatric is significantly higher than that of non-elderly people[5]. Geriatric spend more than $50 \%$ of total curative expenditure[6]. This poses challenges for medical financing.

From the perspective of the cost of the disease distribution, the treatment costs of the population after the age of 60 are mainly used for the treatment of chronic diseases. A study based on the degree of change in results showed that from 2013 to 2017, the proportion of medicine costs accounted for the highest proportion of medical expenses in hospitalization[7]. The average medicine cost and proportion of the geriatric in different medical and health institutions are generally higher than the average of all ages[8]. In order to reduce the burden of patients on medicine costs, in 2017, China implemented a zero-plus drug policy for public hospitals[9]. Although the cost burden of patients was reduced to a certain extent, the effect was not obvious[10]. How to reduce the medical burden on patients has always been an important issue in the field of health care.

In recent years, the role of traditional Chinese medicine(TCM) in the prevention and treatment of diseases has been further highlighted and widely recognized by the international community[11]. Lu's research found that the clinical cure rate of the TCM group (81.8\%) was higher than that of the western medicine group (54.8\%) when treating H1N1[12]. In addition, researchers have found that TCM has a cost advantage in treating certain diseases. Tsai's research shown that the cumulative outpatient medical cost of in the TCM users with heart failure was lower than that of the non-TCM users within 1 year, and the hospitalization cost at 1-year follow-up was lower for TCM users than for non-TCM users[13]. For the treatment of uterine fibroids, the total fibroid-related conventional western medicine consumed by TCM users was less than that by nonusers,the total annual medical cost was less in TCM users than in nonusers[14].

Previous research has shown that the geriatric is more inclined to choose TCM for treatment[15], and the treatment costs of geriatric are mainly used for chronic diseases[16]. The objective of this study was to explore the utilization of TCM in geriatric patients with chronic diseases and the impact of the use of TCM on their medical burden, and analyse which chronic diseases mainly treated with TCM has high cost-effectiveness.

\section{Methods}

\section{Data collection}

The basic data used in this study were obtained from the information system of the sample institutions.The multi-stage stratified sampling method was used to determine the sample institutions. In the first stage, Changsha, Yueyang, Zhuzhou, Yongzhou and Hengyang were selected as sample areas based on the comprehensive consideration of the economic development, demographic characteristics, medical and health conditions, and cultural differences. The second stage was to select one district and three counties from the monitoring cities. The third stage was to choose medical institutions in the monitoring areas at all 
levels according to the type of institutions of administrations structure. The sample data were obtained from 76 hospitals, 36 community health service centers, 102 township hospitals.

\section{Study sample}

The inpatient curative care data of patients over $60 \mathrm{y}$ were derived from the information system of the sample institutions, involving age, gender, disease, length of stay, total and detailed costs of treatment, types of insurance coverage, insurance reimbursement, and out-of-payments(OOPs) of patients in 2018, but did not contain any information that may infer personal identity. Based on the method of disease division in the global disease burden, the remaining diseases are classified as chronic diseases after deducting the acute diseases from the non infectious diseases[17]. After the invalid or false information was excluded in this study, there were 395678 items involving the treatment and nursing expenditure, of which 280929 items of chronic diseases treatment were included (154122 male and 129285 female involved). The age of chronic diseases patients in sample data were from 60 to 107 years old.

\section{Statistical analysis}

The TCM mentioned in this study includes Chinese patent medicine and Chinese herbal medicine. For that the inpatient data were distributed abnormally, Possion regression model was used to analysis the distribution of inpatients who used TCM treatment.Two-sample Kolmogorov-Smirnov test was performed to observe whether there is difference in the expenditure and hospitalization days between TCM users group and TCM nousers group. Spearman correlation analysis was conducted to determine the relationship between proportion of expenses of traditional Chinese medicine to expenses of drugs(Ptcm), total expenditure of hospitalization(Totalexp), OOPs, length of stay. The statistical software STATA 15.0 was used for the analyses. The significance for all statistical tests was indicated by a two-tailed p_value of 0.05 .

\section{Results}

\section{Utilization of TCM in different group of geriatric inpatients}

Among the 280929 geriatric patients with chronic diseases, the proportion of patients in TCM users group and TCM nousers group was $78.64 \%$ and $21.36 \%$ respectively. The results of Poisson regression shown that from the perspective of the treatment probability of TCM, female $(R R=1.009, R R 95 \% \mathrm{Cl}: 1.006-$ 1.013) were slightly higher than male, and those who have not undergone surgery $(1.246,1.237-1.255)$ were higher than those who have undergone surgery. With reference to general hospitals, other types of medical institutions were more likely to use TCM for treatment (all $R R>1$ ). Partial regression coefficients for urban employees, urban and rural residents, work injury insurance, public medical insurance, and other medical insurance types were statistically significant compared to the group of self-paying. Patients in these groups were more likely to experience TCM treatment (Table 1).

Table 1 Poisson regression analysis for utilization of TCM in different group of geriatric inpatients 


\begin{tabular}{|c|c|c|c|c|c|c|c|c|c|c|c|}
\hline & \multicolumn{2}{|c|}{ TCM nouser } & \multicolumn{2}{|c|}{ TCM user } & \multirow[t]{2}{*}{$b$} & \multirow[t]{2}{*}{$s_{b}$} & \multirow[t]{2}{*}{$z$} & \multirow[t]{2}{*}{$p$} & \multirow[t]{2}{*}{$R R$} & \multicolumn{2}{|c|}{$R R 95 \% \mathrm{Cl}$} \\
\hline & $\mathbf{N}$ & $\%$ & $\mathbf{N}$ & $\%$ & & & & & & & \\
\hline & 60014 & 21.36 & 220915 & 78.64 & & & & & & & \\
\hline \multicolumn{12}{|l|}{ Gender } \\
\hline male & 33220 & 55.35 & 119161 & 53.94 & & & & & 1 & & \\
\hline female & 26794 & 44.65 & 101754 & 46.06 & 0.009 & 0.002 & 4.930 & 0.000 & 1.009 & 1.006 & 1.013 \\
\hline \multicolumn{12}{|l|}{ Surgery } \\
\hline Yes & 45554 & 75.91 & 195278 & 88.40 & & & & & 1 & & \\
\hline No & 14460 & 24.09 & 25637 & 11.60 & 0.220 & 0.005 & 58.650 & 0.000 & 1.246 & 1.237 & 1.255 \\
\hline \multicolumn{12}{|l|}{ Age } \\
\hline $60-69$ & 29931 & 49.87 & 99167 & 44.89 & & & & & 1 & & \\
\hline $70-79$ & 20226 & 33.70 & 78069 & 35.34 & 0.017 & 0.002 & 7.820 & $<0.001$ & 1.017 & 1.012 & 1.021 \\
\hline $80-89$ & 9108 & 15.18 & 40241 & 18.22 & 0.028 & 0.003 & 11.180 & $<0.001$ & 1.029 & 1.024 & 1.034 \\
\hline$\geqq 90$ & 749 & 1.25 & 3438 & 1.56 & 0.023 & 0.007 & 3.200 & 0.001 & 1.023 & 1.009 & 1.037 \\
\hline \multicolumn{12}{|l|}{ Institution type } \\
\hline General hospital & 42234 & 70.37 & 98895 & 44.77 & & & & & 1 & & \\
\hline Traditional Chinese medicine hospital & 6578 & 10.96 & 86128 & 38.99 & 0.250 & 0.003 & 127.270 & $<0.001$ & 1.285 & 1.280 & 1.290 \\
\hline Maternity and child care hospital & 114 & 0.19 & 631 & 0.29 & 0.270 & 0.021 & 16.910 & $<0.001$ & 1.311 & 1.270 & 1.352 \\
\hline Community Health Centre & 494 & 0.82 & 1728 & 0.78 & 0.039 & 0.012 & 3.440 & 0.001 & 1.040 & 1.017 & 1.063 \\
\hline Township Hospital & 10594 & 17.65 & 33533 & 15.18 & 0.025 & 0.003 & 7.810 & $<0.001$ & 1.026 & 1.019 & 1.032 \\
\hline \multicolumn{12}{|l|}{ Insurance status } \\
\hline Self-paying & 4633 & 7.72 & 11274 & 5.10 & & & & & 1 & & \\
\hline Commercial health insurance & 129 & 0.21 & 254 & 0.11 & 0.047 & 0.036 & 1.340 & 0.179 & 1.048 & 0.979 & 1.122 \\
\hline Insurance for urban and rural residents & 44104 & 73.49 & 164158 & 74.31 & 0.117 & 0.005 & 24.510 & $<0.001$ & 1.124 & 1.114 & 1.135 \\
\hline Public medical insurance & 2 & 0.00 & 71 & 0.03 & 0.187 & 0.030 & 7.610 & $<0.001$ & 1.206 & 1.149 & 1.265 \\
\hline Employment injury insurance & 4 & 0.01 & 38 & 0.02 & 0.159 & 0.050 & 3.710 & $<0.001$ & 1.172 & 1.078 & 1.274 \\
\hline Other insurance & 141 & 0.23 & 4783 & 2.17 & 0.157 & 0.006 & 29.730 & $<0.001$ & 1.170 & 1.158 & 1.182 \\
\hline Retired cadre insurance & 2329 & 3.88 & 4247 & 1.92 & -0.001 & 0.010 & -0.140 & 0.890 & 0.999 & 0.979 & 1.019 \\
\hline Urban employee insurance & 7990 & 13.31 & 34163 & 15.46 & 0.115 & 0.006 & 22.410 & $<0.001$ & 1.122 & 1.110 & 1.133 \\
\hline Medical assistance & 682 & 1.14 & 1927 & 0.87 & 0.014 & 0.012 & 1.210 & 0.225 & 1.014 & 0.991 & 1.038 \\
\hline
\end{tabular}

\section{Comparison of the cost in TCM users group and TCM nousers group}

According to international Classification of Diseases, Tenth Revision(ICD-10), chronic diseases were classified into 15 categories. The Kolmogorov-Smirnov test was performed on the distribution of the Totalexp, length of stay, medicine cost,OOPs in the TCM nousers group and TCM users group. The results showed that the distribution of Totalexp in the TCM users group and the TCM nousers group was different in various types of diseases (all $p<0.001$ ); the Totalexp of the TCM nousers group was lower than that of TCM users group. TCM nousers group and TCM users group had different distribution in cost of medicine, and the difference was statistically significant (all $p<0.001$ ). Except for digestive diseases, the cost of medicine in the TCM nousers group was lower than that in the TCM users group(Table2). Excluding congenital malformations, deformities, and chromosomal abnormalities $(p=0.059)$, other chronic diseases had different distributions of length of stay in the TCM nousers group and TCM users group $(p<0.05)$. Among patients with various diseases,the distribution of patient's OOPs in the TCM nousers group and TCM users group was different, and the difference was statistically significant (all $p<0.05$ ), the OOPs for patients in the TCM nousers group was higher than that in the TCM users group (Table3).

\section{The effect of using TCM on the cost and burden of geriatric inpatients}

Divide the cost of TCM by the cost of medicine to get the proportion of TCM(Ptcm). A correlation analysis was conducted to find the intercorrelations among Ptcm and Totalexp, cost of medicine,length of stay, OOPs. The results of Spearman correlation test were shown in Table 3. There was no correlation between the Ptcm and Totalexp, length of stay, and OOPs for congenital malformations, deformations and chromosomal abnormalities. The Ptcm correlated significantly and positively with the length of stay $\left(r_{s}>0, p<0.05\right)$, and negatively with the OOPs $\left(r_{s}<0, p<0.001\right)$ for patients with other types diseases. The Ptcm was associated with increased Totalexp for neoplasms, diseases of the blood and blood-farming organs and certain disorders involving the immune 
mechanism, mental and behavioural disorders $\left(r_{s}>0, p<0.001\right)$. The Ptcm was negatively correlated with the Totalexp for Endocrine,nutritional and metabolic diseases, diseases of the nervous system, the ear and mastoid process system, the circulatory system,the respiratory system,the digestive system,the musculoskeletal system and connective tissue, the genitourinary system. There was negative correlation between Ptcm and the cost of medicines for diseases of the nervous system,the ear and mastoid process, the respiratory system,the digestive system,the musculoskeletal system and connective tissue $\left(r_{s}<0\right.$, $p<0.05)$. For other types of diseases, the Ptcm was positively related to the cost of medicine $\left(r_{s}>0, p<0.001\right)($ Table4).

Table2 Two-sample Kolmogorov Smirnov test of Totalexp, medicine cost in TCM nousers group and TCM users group

\begin{tabular}{|c|c|c|c|c|c|c|c|c|}
\hline & \multicolumn{2}{|l|}{$\mathbf{N}$} & \multicolumn{4}{|l|}{ Totalexp } & \multicolumn{2}{|l|}{ Medicine cost } \\
\hline & & & Median $\otimes P 25, P 75 \rrbracket(R M B)$ & & $D$ & $p$ & Median $₫ \mathrm{P} 25, \mathrm{P} 75 \rrbracket(\mathrm{RMB})$ & \\
\hline & $\begin{array}{l}\text { TCM } \\
\text { nouser }\end{array}$ & $\begin{array}{l}\text { TCM } \\
\text { user }\end{array}$ & TCM nouser & TCM user & & & TCM nouser & $\mathrm{Tl}$ \\
\hline Neoplasms & 7028 & 17627 & 4720.93(3099.34,6927.30) & $\begin{array}{l}6748.95 \\
\square 4548.57,9832.43 \rrbracket\end{array}$ & 0.242 & $<0.001$ & $1446.22(517.45,3028.82)$ & $2 \varsigma$ \\
\hline $\begin{array}{l}\text { Diseases of the } \\
\text { blood and blood- } \\
\text { farming organs and } \\
\text { certain disorders } \\
\text { involving the } \\
\text { immune mechanism }\end{array}$ & 536 & 1066 & $4445.67(2890.36,6416.17)$ & $\begin{array}{l}5702.43 \\
\square 3810.53,8254.89 \rrbracket\end{array}$ & 0.199 & $<0.001$ & 728.12(310.80,1387.59) & 16 \\
\hline $\begin{array}{l}\text { Endocrine,nutritional } \\
\text { and metabolic } \\
\text { diseases }\end{array}$ & 2912 & 11551 & $5140.85(3067.33,7263.48)$ & $\begin{array}{l}5758.11 \\
\varangle 3889.28,7985.43 \rrbracket\end{array}$ & 0.108 & $<0.001$ & $1130.68(558.26,1918.92)$ & $1 \varepsilon$ \\
\hline $\begin{array}{l}\text { Mental and } \\
\text { behavioural } \\
\text { disorders }\end{array}$ & 269 & 813 & $4769.90(3005.57,7132.37)$ & $\begin{array}{l}5910.01 \\
\text { \3879.00,8236.55区 }\end{array}$ & 0.153 & $<0.001$ & $740.64(288.28,1566.24)$ & 1 \\
\hline $\begin{array}{l}\text { Diseases of the } \\
\text { nervous system }\end{array}$ & 1511 & 9983 & $4530.85(2735.04,6530.29)$ & 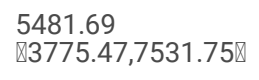 & 0.149 & $<0.001$ & $1022.72(482.65,1902.26)$ & $1 \epsilon$ \\
\hline $\begin{array}{l}\text { Diseases of the eye } \\
\text { and adnexa }\end{array}$ & 4956 & 4312 & $4428.33(2956.06,5750.32)$ & 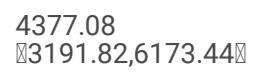 & 0.063 & $<0.001$ & $279.26(142.80,477.13)$ & $9 ;$ \\
\hline $\begin{array}{l}\text { Diseases of the ear } \\
\text { and mastoid } \\
\text { process }\end{array}$ & 355 & 2228 & $2772.94(1430.40,4546.36)$ & $\begin{array}{l}3194.94 \\
\mathbb{Q} 1674.31,5182.72 \rrbracket\end{array}$ & 0.091 & $<0.001$ & $558.58(304.95,1145.56)$ & 96 \\
\hline $\begin{array}{l}\text { Diseases of the } \\
\text { circulatory system }\end{array}$ & 13628 & 72722 & $4069.16(2241.65,6558.29)$ &  & 0.171 & $<0.001$ & $991.62(445.48,1867.33)$ & $1 \varepsilon$ \\
\hline $\begin{array}{l}\text { Diseases of the } \\
\text { respiratory system }\end{array}$ & 7752 & 32866 & $3459.37(1541.86,6072.20)$ & $\begin{array}{l}4744.12 \\
\square 2375.2,7351.23 \square\end{array}$ & 0.142 & $<0.001$ & $954.97(398.12,1914.56)$ & 16 \\
\hline $\begin{array}{l}\text { Diseases of the } \\
\text { digestive system }\end{array}$ & 10490 & 20269 & $4322.13(2358.77,6664.52)$ & 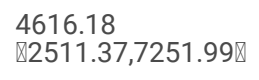 & 0.050 & $<0.001$ & $1031.51(466.85,1943.96)$ & $1:$ \\
\hline $\begin{array}{l}\text { Diseases of the skin } \\
\text { and subcutaneous } \\
\text { tissue }\end{array}$ & 1070 & 2647 & $2362.60(1198.11,4251.12)$ & 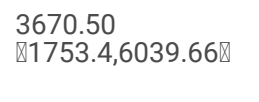 & 0.201 & $<0.001$ & $531.44(211.19,1206.53)$ & $1 s$ \\
\hline $\begin{array}{l}\text { Diseases of the } \\
\text { musculoskeletal } \\
\text { system and } \\
\text { connective tissue }\end{array}$ & 3960 & 31597 & $2292.09(984.83,4914.41)$ & 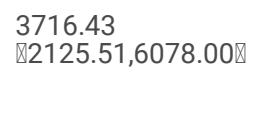 & 0.251 & $<0.001$ & $424.31(179.50,950.09)$ & 91 \\
\hline $\begin{array}{l}\text { Diseases of the } \\
\text { genitourinary } \\
\text { system }\end{array}$ & 5427 & 12877 & $3810.23(2081.08,6554.31)$ & $\begin{array}{l}5202.00 \\
\bigotimes 2826.17,8575.96 \rrbracket\end{array}$ & 0.150 & $<0.001$ & $820.63(363.85,1622.19)$ & 1 ? \\
\hline $\begin{array}{l}\text { Congenital } \\
\text { malformations, } \\
\text { deformations and } \\
\text { chromosomal } \\
\text { abnormalities }\end{array}$ & 120 & 357 & $4824.48(3060.58,7750.71)$ & $\begin{array}{l}6259.02 \\
\square 4694.00,8010.26 \rrbracket\end{array}$ & 0.248 & $<0.001$ & $1072.035(491.29,2160.54)$ & $2 c$ \\
\hline
\end{tabular}


Table3 Two-sample Kolmogorov Smirnov test of length of stay, OOPs in TCM nousers group and TCM users group

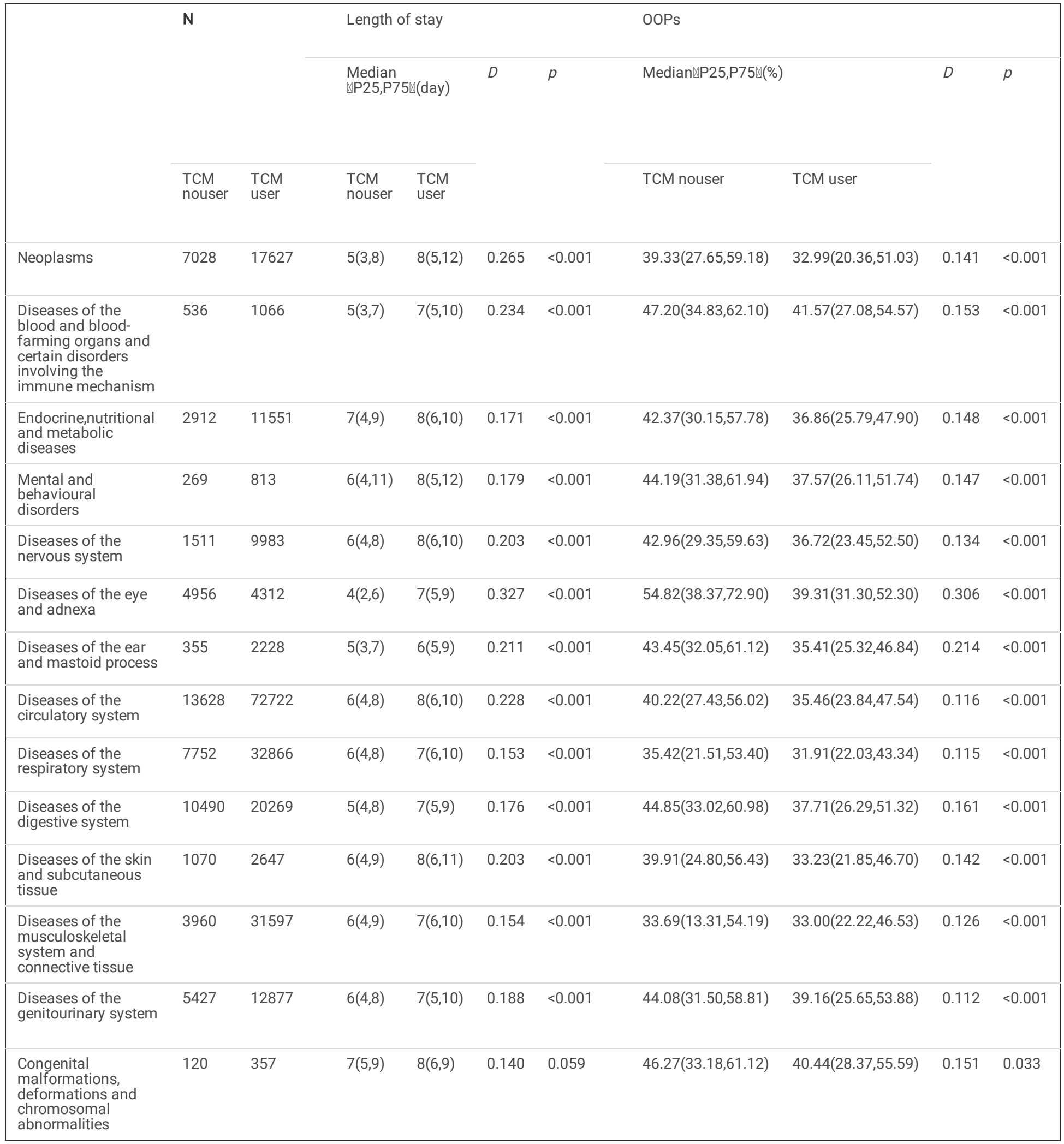

Table4 Spearman correlation analysis among Ptcm, Totalexp, medicine cost, length of stay, OOPs 


\begin{tabular}{|c|c|c|c|c|c|c|c|c|}
\hline & \multicolumn{2}{|c|}{ Totalexp } & \multicolumn{2}{|c|}{ Medicine cost } & \multicolumn{2}{|c|}{ Length of stay } & \multicolumn{2}{|l|}{ OOPs } \\
\hline & $r_{s}$ & $p$ & $r_{s}$ & $p$ & $r_{s}$ & $p$ & $r_{s}$ & $p$ \\
\hline \multicolumn{9}{|l|}{ Ptcm of various diseases } \\
\hline Neoplasms & 0.185 & $<0.001$ & 0.217 & $<0.001$ & 0.204 & $<0.001$ & -0.116 & $<0.001$ \\
\hline $\begin{array}{l}\text { Diseases of the blood and blood-farming organs and certain } \\
\text { disorders involving the immune mechanism }\end{array}$ & 0.067 & 0.008 & 0.229 & $<0.001$ & 0.139 & $<0.001$ & -0.165 & $<0.001$ \\
\hline Endocrine,nutritional and metabolic diseases & -0.082 & $<0.001$ & 0.073 & $<0.001$ & 0.090 & $<0.001$ & -0.141 & $<0.001$ \\
\hline Mental and behavioural disorders & 0.077 & 0.011 & 0.235 & $<0.001$ & 0.121 & $<0.001$ & -0.122 & $<0.001$ \\
\hline Diseases of the nervous system & -0.068 & $<0.001$ & -0.038 & $<0.001$ & 0.143 & $<0.001$ & -0.145 & $<0.001$ \\
\hline Diseases of the eye and adnexa & -0.018 & 0.079 & 0.376 & $<0.001$ & 0.302 & $<0.001$ & -0.288 & $<0.001$ \\
\hline Diseases of the ear and mastoid process & -0.197 & $<0.001$ & -0.052 & 0.008 & 0.055 & 0.005 & -0.276 & $<0.001$ \\
\hline Diseases of the circulatory system & -0.052 & $<0.001$ & 0.024 & $<0.001$ & 0.142 & $<0.001$ & -0.144 & $<0.001$ \\
\hline Diseases of the respiratory system & -0.132 & $<0.001$ & -0.043 & $<0.001$ & 0.021 & $<0.001$ & -0.134 & $<0.001$ \\
\hline Diseases of the digestive system & -0.123 & $<0.001$ & -0.012 & 0.031 & 0.126 & $<0.001$ & -0.212 & $<0.001$ \\
\hline Diseases of the skin and subcutaneous tissue & 0.024 & 0.139 & 0.156 & $<0.001$ & 0.109 & $<0.001$ & -0.153 & $<0.001$ \\
\hline $\begin{array}{l}\text { Diseases of the musculoskeletal system and connective } \\
\text { tissue }\end{array}$ & -0.047 & $<0.001$ & -0.054 & $<0.001$ & 0.029 & $<0.001$ & -0.094 & $<0.001$ \\
\hline Diseases of the genitourinary system & -0.071 & $<0.001$ & 0.040 & $<0.001$ & 0.045 & $<0.001$ & -0.121 & $<0.001$ \\
\hline $\begin{array}{l}\text { Congenital malformations, deformations and chromosomal } \\
\text { abnormalities }\end{array}$ & -0.037 & 0.421 & 0.112 & 0.014 & 0.037 & 0.419 & -0.081 & 0.079 \\
\hline
\end{tabular}

\section{Discussion}

The choice of treatment mode is related to the patient, sometimes influenced by the doctor and the type of disease. Regarding the situation of using TCM in different groups of geriatric inpatients with chronic diseases, our results indicate that the possibility of TCM treatment in female inpatients with chronic diseases was higher than that in male. On the one hand, it may be affected by the patient's preference for medical treatment. In 2016 , the results of the national survey on health care literacy of TCM shown that female pay more attention to health care issues of TCM in life than male[18]. Chung's study found that women were more likely to choose TCM for treatment of diseases[19]. On the other hand, it may be related to the distribution of dominant diseases in TCM. According to the survey of the predominant diseases of TCM, the predominant diseases of TCM are distributed in internal, external, gynecology, pediatrics, orthopedics, ophthalmology, otolaryngology[20]. If female with chronic diseases suffer from gynecological diseases at the same time, doctors are more likely to choose TCM for treatment.

Our study also found that taking the group of $60-69 \mathrm{y}$ as a reference, the elderly were more likely to receive TCM treatment. This may be related to the trust of TCM. The affirmation and trust of the efficacy of TCM is the key factor affecting the residents' consumption behavior of TCM[21]. Qiu's research shown that age has the greatest influence on the factors of trust and seeking medical treatment $\$ and the elderly have greater trust in TCM, and they are more likely to use TCM for treatment[22]. In our study, patients who have undergone surgery were less likely to use TCM. This may be the reason that surgical patients must be fasting before and after surgery, and also can not take oral TCM.

From the comparison of various institutions, the possibility of TCM treatment in general hospitals was lower than other institutions. Differences in the allocation of TCM resources among different types of institutions may lead to this situation. Cai's results shown that the number of TCM doctors was an important factor affecting the provision of TCM services by institutions[23]. In 2018, the proportion of TCM physicians in general hospitals (7.23\%) in Hunan province was lower than in TCM hospitals (48.11\%), community health service centers (18.28\%), and township health centers (14.18\%). Western doctor does not have the legal prescription right of Chinese herbal medicine, so the utilization rate of TCM was lower in general hospitals with less doctors of TCM.

In our study, compared with the self-financed patient group, the possibility of TCM treatment in groups of urban employees, urban and rural residents, work injury insurance, public health care, and other medical insurance types were higher.This may be the effect of medical insurance on the compensation of TCM services. Patients with chronic diseases who need to be hospitalized are in serious condition. Some studies have shown that under the hypothesis of severe chronic disease, patients are more inclined to choose western medical services and willingness to pay low-cost medical treatment[24]. In recent years, China has formulated a series of policies and measures to promote the role of TCM in disease treatment. The Chinese medicine law of the people's Republic of China, which came into effect in 2017, clearly stipulates that governments at or above the county level should include eligible TCM decoction pieces, proprietary TCM, and TCM preparations in medical institutions into the basic medical insurance fund payment scope[25]. At present, there are 1,321 kinds of Chinese patent medicines, and 892 kinds of TCM decoction pieces have been included in the "National Basic Medical Insurance, Work Injury Insurance and Maternity Insurance Drug Catalogue"[26]. Medical insurance not only increases the willingness of patients to use TCM services through economic means, but also increases the enthusiasm of medical institutions to provide TCM services. 
The length of stay, the cost of medicine, Totalexp, and OOPs are often used as indicators to evaluate the patient's hospitalization burden[27][28][29]. Ji Conghua's study concluded that patients using TCM were higher than the western medicine group in terms of length of stay, total cost of hospitalization of medicines, etc[30]. In our study, we found that for geriatric inpatients with chronic congenital malformations, deformities, and chromosomal abnormalities, the difference in hospitalization days between the group of TCM user and the TCM nousers group was not significant. For digestive diseases, the cost of medicines in the TCM users group was lower than that in the TCM nousers group. For patients with different types of diseases and symptoms, the means of TCM treatment of digestive system diseases are not necessarily all drug treatment, but also Taiji, dietotherapy, acupuncture, massage, which may explain why the drug cost of TCM nousers group was lower[31]. Although the Totalexp level of the TCM users group for most diseases were higher than those of TCM nousers group, the OOPs in TCM users group was lower than that of TCM nousers group. This may be related to the health insurance reimbursement policy. When the national basic medical insurance pays the expenses of Chinese patent medicine and Western medicine, it is divided into class $A$ and class $B$. The expenses incurred by using class a drugs and Chinese herbal pieces included in the medical insurance shall be paid in full by the medical insurance fund[32]. Among the drugs included in the drug catalog of national basic medical insurance, 1134 kinds (51.24\%) of TCM and 398 kinds ( $30.11 \%$ ) of Western medicine can be fully paid by the fund. Only less than 40 kinds of Chinese herbal pieces have not been included in the scope of fund payment. The number of Western medicines not included in the fund is far more than that.

Regarding the relationship between the use of TCM treatment and the length of stay, previous research conclusions are not completely consistent. The results of Wang's study showed that the length of stay with TCM treatment was longer[33]. Li found that the combined treatment of ischemic stroke with TCM and west medicine can reduce the length of stay[34]. Our results shown that the Ptcm was negatively related to the length of stay for inpatient treatment of various chronic diseases for geriatric. We did not distinguish between specific diseases, so we are not sure whether there is such a correlation between the Ptcm and the length of stay for each disease. It should be noted that the severity of the illness is the most important factor affecting the length of stay[35]. Since we did not obtain information about the condition, we cannot rule out the increase in the Ptcm caused by the degree of the disease, which also affected the length of stay.

Concerning the impact of Ptcm on the cost of medicines and Totalexp, previous studies have shown that the cost of medicines decreases as Ptcm increases[36]. The higher Ptcm, the lower Totalexp[37]. We have found that Ptcm was not necessarily related to Totalexp of geriatric inpatients with chronic diseases. For certain diseases, the cost of medicines and Totalexp may increase with the increase in Ptcm, such as tumors, blood and hematopoietic organ diseases and certain diseases involving immune mechanisms, endocrine, nutritional and metabolic diseases, mental and behavioral disorders, eye and appendage disorders, urogenital diseases. On the one hand, it may be determined by the natural characteristics of the disease species. Taking geriatric inpatients with cancer as an example, their disease severity is relatively high. Many patients were treated with TCM for symptomatic treatment in the later stage because of unsatisfactory effect after previous repeated treatment. The treatment characteristics of the disease are long hospital stays, high proportion of drugs, and high total cost of a single hospital stay. On the other hand, it may be affected by the degree of illness. Some patients are hospitalized in critical condition. Doctors often choose west medicine such as infusion to control their condition at first[38]. After patient's condition improves, TCM could be need to take for a long time. Although the cost of hospitalization of certain diseases will increase with the increase of Ptcm, Ptcm was negatively related to OOPs. It shows that the higher Ptcm, the smaller the burden on patients.

According to the relationship between Ptcm for various diseases, the cost of medicines, and the cost of hospitalization,We believe that the use of TCM for the treatment of geriatric chronic diseases respiratory system, digestive system, ear diseases, nervous system are relatively cost-effective. The higher the Ptcm, the lower the cost of medicines and Totalexp, and the lower OOPs. The use of TCM for the treatment of chronic circulatory diseases and ophthalmic diseases, although the cost of medicine is higher, but Totalexp and the OOPs decrease with the increase of Ptcm.

To our knowledge, this is the first comprehensive report on the use and cost of TCM in geriatric inpatients with chronic diseases. In this study, not only the situation of using TCM were analyzed from the perspective of gender, age, institution, and insurance status, but also the relationship between the Ptcm and the cost of hospitalization and personal burden of various geriatric chronic patients were discussed. However, It is undeniable that our research still has two limitations. First, the data we obtained only reflects a single cost and cannot reflect the annual cost and burden level of geriatric inpatients with chronic diseases. In addition, due to space limitations, there is a lack of research on the path of Ptcm affects hospitalization costs.

\section{Conclusion}

There are differences in the distribution of TCM treatment of geriatric inpatients with chronic diseases in terms of gender, age, institution, and insurance status. Treatment mainly used TCM for geriatric chronic respiratory system, consumption system diseases, circulatory system, ear diseases and neurological diseases has obvious cost-benefit advantages, and conducive to reducing the burden of patients.

\section{Abbreviations}

TCM: traditional Chinese medicine

Totalexp:the total hospitalization expenses

OOPs:out-of-pocket payments;

Ptcm: proportion of expenses of traditional Chinese medicine to expenses of drugs

\section{Declarations}




\section{Acknowledgments}

The authors express thanks to Health Commission of Hunan Province for their the support on the data collection.

\section{Funding}

The study was supported by Natural Science Foundation of China(NSDC)(71673299),Natural Science Fund general project of Hunan(NSFH) (2019JJ40213),Scientific Research Project of Hunan Provincial Department of Education(PHDE)(18C0363),Project entrusted by Health Commission of Hunan(PHCH)(WTB201902). The NSFC,NSFH funded travel to the data collection. The PHDEXPHCH funded computer equipment for research $\square$

\section{Availability of data and materials}

The datasets generated and/or analyzed during the current study are available from the corresponding author on reasonable request.

\section{Authors' contributions}

$\mathrm{HZ}$ drafted the manuscript and performed the statistical analysis.ZS, LJ did some expeditionary research. LRZ,XW helped revise the manuscript. All authors read and approved the final manuscript.

\section{Ethics approval and consent to participate}

The study was approved by Ethics Committee of The First Hospital of Hunan University of Chinese Medicine (HN-LL-KY-2019-18). All participants provided verbal consent prior to participation in the study. This method of obtaining consent was approved by the ethics committee as the study presented no more than minimal risk to the participants.

\section{Consent for publication}

Not Applicable.

\section{Competing interests}

The authors declare that they have no competing interests.

\section{Authors' Information}

1.School of Humanities and Management, Hunan University of Chinese Medicine,

Changsha, Hunan Province, China(PRC) .

2.School of Nursing, China Medical University, Shenyang, Liaoning Province, China(PRC).

3.Humanity and Management College, Shaanxi University of Chinese Medicine, Xianyang, Shaanxi, China (PRC).

4.College of the Humanities and Social Sciences, China Medical University, Shenyang, Liaoning Province, China (PRC).

\section{References}

1. National Bureau of Statistics of China:Population growth slows down and urbanization continues to improve,http://www.stats.gov.cn/tjsj/sjjd/202001/t20200119_1723861.html. Accessed 21 Jan 2020.

2. Fulmer T. A special report: The World Assembly on Aging. Journal of Gerontological Nursing.1982,8(12). doi:10.3928/0098-9134-19821201-09

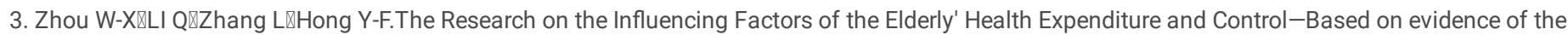
Elderly' Questionnaire in An Hui Province.China geriatric health medicine.2017; doi:10. 3969 /j. issn.1672ه4860. 2017. 06. 017.

4. Li F, Di L-L, Wang L-N, Zhang X-X, Wang C-Y,Chen W, Cui X, Shao Z-Y, Jin C-L.Analysis on the demand, utilization and cost characteristics of medical service for the elderly in Shanghai.Health economics research,2018(06):26-29. doi:10.14055/j.cnki.33-1056/f.20180529.013

5. Zheng J-P, Ma L, Xu Y, Peng Y-H, Cao J-J, Lei J, Xia J. Inpatient status and expenses of medical insurance for the elderly in a top three hospital of Xinjiang corps. Chinese Journal of Gerontology.2017,37(18):4646-4648. doi: 10. 3969 /j. issn.1005-9202.2017.18.102.

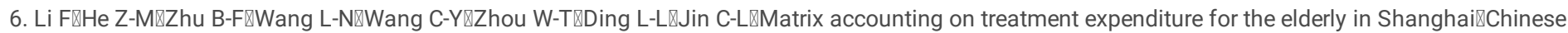
Health Resources.2018囚12(4):285-290. doi:10.13688/j.cnki.chr.2018.17884.

7. Zhang Y, Jiang Q, Yang X-Y, et al,Research on the Structure Variation of Hospitalization Expenses in a Tertiary-level Hospitals Based on Structure Changing Degree.Chinese Health Economics, 2019,38(9):69-71. doi:10.7664/CHE20190918

8. Wang L-N,Ding L-L,Zhu B-F,Wang C-Y,Li F,Jin C-L.Comparative research on drug expenditure for the elderly in Shanghai.Chinese Health Resources.2019,22(2). doi:10.13688/j.cnki.chr.2019.18313.

9. Health and Health Commission of the people's Republic of China,Notice on comprehensively promoting the comprehensive reform of public hospitals. http://www.nhc.gov.cn/tigs/s3581/201704/0563e06eff4441ffa9772dc30b487848.shtml. Accessed 21 Jan 2020. 
10. Xu L,Zheng E-Wi,Based on the Perspective of Hospitalized Cases to Analysis the Effect of Zero Price Addition Policy on the Hospitalization Expense. Chinese Medicine Record. 2019. 20(6):33-35.

11. National Administration of Traditional Chinese Medicine,Outline of strategic plan for development of traditional Chinese medicine (2016-2030), http://bgs.satcm.gov.cn/zhengcewenjian/2018-03-24/865.html. Accessed 21 Jan 2020.

12. Lu C, Lu Y, Zhou H. et al. A preliminary study on the medical expenditure of Chinese medicine and integrative medicine treatment for influenza a (H1N1) in the fever clinics.Chin. J. Integr. Med. 2010,16: 493.https://doi.org/10.1007/s11655-010-0563-4

13. Tsai MY, Hu WL, Chiang JH, et al. Improved medical expenditure and survival with integration of traditional Chinese medicine treatment in patients with heart failure: A nationwide population-based cohort study. Oncotarget. 2017;8(52):90465-90476. doi:10.18632/oncotarget.20063

14. Su SY, Muo CH, Morisky DE. Use of Chinese medicine correlates negatively with the consumption of conventional medicine and medical cost in patients with uterine fibroids: a population-based retrospective cohort study in Taiwan. BMC Complement Altern Med. 2015;15:129. doi:10.1186/s12906-015-06450

15. Chung VCh, Lau CH, Yeoh EK, Griffiths SM. Age, chronic non-communicable disease and choice of traditional Chinese and western medicine outpatient services in a Chinese population. BMC Health Serv Res. 2009,9:207. doi:10.1186/1472-6963-9-207

16. Zhang Y-H, Wan Q, Chai P-P, et al,The Accounting Results of China Current Health Expenditure Based on SHA 2011.Chinese Health Economics.2015,34(3):12-16.doi:10.7664/CHE20150303

17. Li Z-Y, Li B, Zhang Y-H, et al. Study on the System of Traditional Chinese Medicine Expenditure Accounts on Non -communicable Disease Prevention and Treatment.Chinese Health Economics.2012,31(5):5-9.

18. Yang L. Results of 2016 national survey on health and health literacy of traditional Chinese medicine. Management of traditional Chinese medicine. 2016,24 (08): 104

19. Chung VCh, Lau CH, Yeoh EK, Griffiths SM. Age, chronic non-communicable disease and choice of traditional Chinese and western medicine outpatient services in a Chinese population. BMC Health Serv Res. 2009;9:207. doi:10.1186/1472-6963-9-207

20. Yang H-F, Cui Z-W, Wang M, Zhao Z. Distribution and characteristics of dominant diseases of traditional Chinese medicine in China. Journal of traditional Chinese medicine. 2012,53 (04): 285-287

21. Chen $Y-X$. Study on the influencing factors of Chinese herbal medicine consumer behavior . Fujian agricultural and Forestry University, 2012

22. Qiu H-Z, Liang R-Q, Huang G-X, Dou X-Y, Yuan X-Q, et al. A study on the influence of trust on people's use of TCM service behavior. Journal of .TCM management.2010,18 (10): 865-868. doi:10.16690/j.cnki .1007-9203.2010.10.004

23. Cai $Y$. Research on influencing factors and needs assessment of TCM service provision. Wuhan University, 2013

24. Peng Y-Y, Xiong J-Y, LI X-L, Jia E-P, Jiang M-Z, Shen X. Study on medical service utilization preference of urban elderly chronic patients based on Dis- crete Choice Experiment . Chinese Journal of Health Policy. 2019,12(9).doi:10.3969 /j. issn.1674-2982. 2019. 09. 010

25. The National People's Congress of the People's Republic of China,The Chinese medicine law of the people's Republic of China, http://www.npc.gov.cn/zgrdw/npc/xinwen/2016-12/25/content_2004972.htm, Accessed 20 Jan 2020.

26. National Healthcare Security Administration. "National Basic Medical Insurance, Work Injury Insurance and Maternity Insurance Drug Catalogue".http://www.nhsa.gov.cn/art/2019/8/20/art_37_1666.html. Accessed 20 Jan 2020.

27. Gussoni G, Foglia E,Frasson S, Casartelli L,Campanini M,Marzia Bonfanti M,et al. Real-world economic burden of venous thromboembolism and antithrombotic prophylaxis in medical inpatients. Thrombosis Research.2013,131(1).doi:10.1016/j.thromres.2012.10.008

28. Spencer-Bonilla, G., Quiñones, A.R., Montori, V.M. Assessing the Burden of Treatment. Journal of general internal medicine.2017,32(10).doi:10.1007/s11606-017-4117-8

29. Xu Z-Y, Zhang M, Cui Y-P. Develoment and Application of Study on Disease Burden.China Cancer 2013,22(8):638-641.doi:10.11735/j.issn.10040242.2013.08.A008

30. Ji C-H. Advantages of TCM Diseases in Health Economics Evaluation,Chinese health economics. 2012,31(11):67-69.

31. Wu F. Research of Chinese medicine treatment for psychosomatic disease of digesting system. Hunan University of TCM.2007.

32. Xue H-Y®Yu Z-Y®Li J.Decipherment and Consideration of 2019 Edition of Medicine List for National Basic Medical Insurance区Employment Injury Insurance and Maternity Insurance. Herald of Medicine.2020,39(1):1-9.doi:10.3870/j.issn.1004-0781.2020.01.001

33. Wang X-F, Liu J-P, Shen K-L, Ma R, Cui Z-Z, Deng L, Shang Y-X, et al. Comparison of living expenses of 1298 children with pneumonia and analysis of the benefits of using traditional Chinese medicine. World science and technology - modernization of traditional Chinese Medicine,World Science and Technology/Modernization of Traditional Chinese Medicine and Materia Medica.2013,15(05):994-1001.doi: 10.11842/wst.2013.05.039

34. Li Y, Xi H-X, Zhu S, Yu N, Wang J, Li Y, Yu G-P, Ma X-M, Zhang J, et al. Cost-Effectiveness Analysis of Combined Chinese Medicine and Western Medicine for Ischemic Stroke Patients. Chinese Journal of Integrative Medicine,2014,20(08):570-584.

35. Li Q. A study on the influencing factors of the average hospitalization expenses and hospitalization days of the patients with three chronic diseases in a general hospital. Jinan: Shandong University, 2015

36. Du J-N, Yin S, Zhao X, et al.The Usage Ratio and the Influencas for the cost of Using Chinese Traditional Medicine on Inpatient $\mathbb{}$ Chinese Primary Health Care $\varangle 2015,29(9): 11-13$. doi:10.3969/j.issn.1001-568X.2015.09.0004

37. Lu Y-L-X,Li L-G,Bian Y. How to reduce health expenses by using Traditional Chinese Medicine( TCM) ? Based on the analysis of inpatients' TCM expenses and typical diseases in Ningxia Province.Chinese Journal of Health Policy,2017,10 (7). doi: 10.3969 /j.issn.1674-2982. 2017. 07. 009. 
38. Chen Y-X, Yu J-H. Analysis of the application of traditional Chinese medicine in inpatients of a hospital in 2010 . Journal of Guiyang College of traditional Chinese medicine, 2012,34 (03): 41-45. doi: 10.3969 /j. issn.1002-1108. 2012. 03. 018. 